\title{
Prolonged sitting may increase diabetes risk in physically inactive individuals: an 11 year follow-up of the HUNT Study, Norway
}

\author{
Bjørn O. Åsvold ${ }^{1,2} \cdot$ Kristian Midthjell $^{1} \cdot$ Steinar Krokstad $^{1} \cdot$ Vegar Rangul $^{1}$ \\ Adrian Bauman ${ }^{3}$
}

Received: 21 October 2016 / Accepted: 14 December 2016 / Published online: 4 January 2017

(C) Springer-Verlag Berlin Heidelberg 2017

\begin{abstract}
Aims/hypothesis We examined the association between sitting time and diabetes incidence, overall and by strata of leisuretime physical activity and BMI.

Methods We followed 28,051 adult participants of the NordTrøndelag Health Study (the HUNT Study), a populationbased study, for diabetes incidence from 1995-1997 to 2006-2008 and estimated HRs of any diabetes by categories of self-reported total daily sitting time at baseline.

Results Of 28,051 participants, 1253 (4.5\%) developed diabetes during 11 years of follow-up. Overall, sitting $\geq 8 \mathrm{~h} /$ day was associated with a $17 \%(95 \%$ CI 2,34$)$ higher risk of developing diabetes compared with sitting $\leq 4 \mathrm{~h} /$ day, adjusted for age, sex and education. However, the association was attenuated to a non-significant $9 \%(95 \% \mathrm{CI}-5,26)$ increase in risk after adjustment for leisure-time physical activity and BMI. The association between sitting time and diabetes risk differed by leisure-time physical activity $\left(p_{\text {Interaction }}=0.01\right)$. Among participants with low leisure-time physical activity ( $\leq 2 \mathrm{~h}$ light activity per week and no vigorous activity), sitting
\end{abstract}

Electronic supplementary material The online version of this article (doi:10.1007/s00125-016-4193-z) contains peer-reviewed but unedited supplementary material, which is available to authorised users.

Bjørn O. Åsvold

bjorn.o.asvold@ntnu.no

1 HUNT Research Centre, Department of Public Health and General Practice, Faculty of Medicine, NTNU, Norwegian University of Science and Technology, Forskningsveien 2, N-7600 Levanger, Norway

2 Department of Endocrinology, St Olavs Hospital, Trondheim University Hospital, Trondheim, Norway

3 School of Public Health, Sydney University, Sydney, NSW, Australia
$5-7 \mathrm{~h} /$ day and $\geq 8 \mathrm{~h} /$ day were associated with a $26 \%(95 \% \mathrm{CI}$ $2,57)$ and $30 \%(95 \%$ CI 5,61$)$ higher risk of diabetes, respectively, compared with sitting $\leq 4 \mathrm{~h} /$ day. There was no corresponding association among participants with high leisuretime physical activity ( $\geq 3 \mathrm{~h}$ light activity or $>0 \mathrm{~h}$ vigorous activity per week). There was no statistical evidence that the association between sitting time and diabetes risk differed by obesity $\left(p_{\text {Interaction }}=0.65\right)$.

Conclusions/interpretation Our findings suggest that total sitting time has little association with diabetes risk in the population as a whole, but prolonged sitting may contribute to an increased diabetes risk among physically inactive people.

Keywords Epidemiology $\cdot$ Sedentary lifestyle $\cdot$ Type 2 diabetes mellitus
Abbreviations
FINDRISC Finnish diabetes risk score
HUNT Study Nord-Trøndelag Health Study

\section{Introduction}

The global epidemic of diabetes requires preventive actions at a population level. Although low physical activity levels are a recognised risk factor for diabetes, recent interest has focused on specific sedentary behaviours ('too much sitting') [1]. Sedentary behaviours comprise prolonged sitting in different settings (at home, at work, watching television and in cars) but are quite distinct from the risk of physical inactivity, which refers to not meeting recommended physical activity guidelines. Sitting time is of interest in diabetes, given the experimental and physiological evidence that prolonged and 
uninterrupted sitting is adversely related to glucose levels and insulin resistance [1].

Epidemiological studies initially showed associations between prolonged television time and increased type 2 diabetes incidence [2-4], with a meta-analysis indicating that the risk of type 2 diabetes increased by $20 \%$ for each $2 \mathrm{~h}$ of television watched per day [3]. Since television time, the most common form of sitting in the domestic setting, is associated with the diabetes risk factors of snacking and other unhealthy dietary habits and low socioeconomic status [4, 5], it is important to consider other dimensions of sitting exposure to assess the independence of any observed epidemiological risk. Emerging studies examining 'total sitting time' and diabetes have failed to find the same relationships as the early studies of television time. Evidence from a Danish 5 year prospective study suggests that the association of total sitting time with risk of any diabetes seems to be attenuated by physical activity and among non-obese individuals, leaving residual risk for developing diabetes among those who are inactive or obese [6]. In an 11 year follow-up of the Nord-Trøndelag Health Study (the HUNT Study) in Norway, we aimed to investigate the association between total sitting time and the risk of any diabetes, and to examine whether this association was modified by leisure-time physical inactivity or obesity.

\section{Methods}

The HUNT Study is a population-based study in NordTrøndelag county, Norway, with three waves of data collection: HUNT1 (1984-1986), HUNT2 (1995-1997) and HUNT3 (2006-2008). At each survey, all residents aged $\geq 20$ years were invited to participate, and participants completed extensive health-related questionnaires and underwent a clinical examination and random blood sampling, as described previously [7] and on the HUNT website (www. ntnu.edu/hunt, accessed 20 November 2016). The present study uses the HUNT2 Survey as the baseline risk factor examination and the HUNT3 Survey 11 years later as the follow-up examination to assess diabetes incidence.

In HUNT2, 93,898 people were invited and 65,229 (69\%) participated. On a questionnaire that was returned at the clinical examination, participants reported whether they had ever been diagnosed with diabetes, their highest education and their weekly average of leisure-time physical activity during the last year, categorised separately for light (not sweaty or breathless) and vigorous activity (sweaty or breathless) as no, $<1,1-2$ or $\geq 3 \mathrm{~h} /$ week. A second questionnaire was given to all participants at the clinical examination and returned by 55,376 $(85 \%)$ by mail in a pre-paid envelope; this included a question on sitting time: 'How many hours do you usually spend in the sitting position during a 24 hour period? (work, meals, television, car etc.)'. The clinical examination included standardised measurements of weight and height. Random serum glucose concentrations were measured using an enzymatic hexokinase method on a Hitachi 911 Autoanalyzer at Levanger Hospital, Nord-Trøndelag Hospital Trust.

In HUNT3, all participants were asked whether they had ever been diagnosed with diabetes and their age at diagnosis, and random serum glucose was measured in all participants. All participants had their FINDRISC (Finnish diabetes risk score) diabetes prediction score calculated, and participants with a FINDRISC $\geq 15$ out of 26 were invited to have fasting serum glucose, 120 min $75 \mathrm{~g}$ OGTT and $\mathrm{HbA}_{1 \mathrm{c}}$ measurements, as previously described [8].

The validity of self-reported diabetes in this population was examined after the HUNT1 Survey by comparison with general practitioners' records, and the self-report had excellent positive $(96 \%)$ and negative $(99.7 \%)$ predictive values. Selfreported diabetes duration was on average 0.4 years longer than physician-verified duration [9]. Assessing the validity of self-reported long-term physical activity and sitting habits is difficult because of the lack of a true reference standard. However, the HUNT2 questionnaire item on vigorous leisuretime physical activity correlated moderately with $\dot{V} \mathrm{O}_{2 \max }$ $(r=0.46)$ and time spent in vigorous activity with metabolic equivalent values $\geq 6$ as measured during a 1 week ActiReg position-and-movement monitoring $(r=0.31)$, whereas weaker or no correlations were observed for self-report of light leisure-time physical activity [10]. Validity of self-reported sitting time has not been examined in the HUNT Study, but the similar International Physical Activity Questionnaire (IPAQ) item on sitting time correlated moderately $(r \sim 0.3)$ with accelerometer counts depicting sedentary behaviour [11].

Among 65,229 participants in HUNT2, we excluded 10,777 individuals aged $\geq 70$ years, as few attended HUNT3 11 years later. Further, we excluded 1024 individuals with previously known diabetes, 144 with probable diabetes at HUNT2 indicated by random serum glucose $\geq 11.1 \mathrm{mmol} / 1,8617$ who did not return the second questionnaire with details on sitting time, 3006 who returned the second questionnaire but did not report sitting time, 56 who did not answer whether they had diabetes and 780 individuals with missing information on education level, leaving 40,825 participants eligible for follow-up. Among them, 28,051 (69\%) attended the follow-up examination at HUNT3 11 years later and were included in our analyses.

Diabetes at HUNT3 was indicated by self-reported diagnosis, random serum glucose $\geq 11.1 \mathrm{mmol} / 1$ or, in participants who attended the additional examination with fasting serum glucose $\geq 7.0 \mathrm{mmol} / 1,120 \mathrm{~min}$ serum glucose $\geq 11.1 \mathrm{mmol} / 1$ in the OGTT or $\mathrm{HbA}_{1 \mathrm{c}} \geq 6.5 \%$ (48 $\mathrm{mmol} / \mathrm{mol}$ ), consistent with cutoff levels recommended by the WHO/International Diabetes Federation. For people whose diabetes was diagnosed between HUNT2 and HUNT3, date of diabetes diagnosis was estimated from the participants' self-reported age at diagnosis. The median time from HUNT2 to self-reported time of diagnosis 
(6.8 years) was assigned to participants who did not self-report their age at diagnosis (7\%). Date of HUNT3 participation was considered as the date of diagnosis for people whose diabetes was diagnosed by glucose or $\mathrm{HbA}_{1 \mathrm{c}}$ measurements in HUNT3.

We examined the association between self-reported daily sitting time $(\leq 4,5-7$ or $\geq 8 \mathrm{~h})$ and the incidence of any diabetes using Cox proportional hazard models, where participants were followed up from their date of participation in HUNT2 until their date of diabetes diagnosis or participation in HUNT3, whichever occurred first. In a first model, we adjusted for age (by using age as the time scale), sex and education $(<10,10-12$ or $>12$ years). We additionally adjusted for baseline levels of BMI (weight in $\mathrm{kg}$ divided by the squared height in $\mathrm{m}$, continuous) and selfreported weekly leisure-time physical activity (categorised as no, low $[<3 \mathrm{~h}$ of light and no vigorous activity], medium $[\geq 3 \mathrm{~h}$ of light or $<1 \mathrm{~h}$ of vigorous activity] or high [ $\geq 1 \mathrm{~h}$ of vigorous activity]), which could either be confounders or proxies for lifestyle/dietary confounders, or be on the causal pathway between sitting time and diabetes. Additionally, we examined the association of sitting time with diabetes risk stratified by leisuretime physical activity (dichotomised as $\leq 2 \mathrm{~h}$ light and no vigorous activity/week vs $\geq 3 \mathrm{~h}$ light or $>0 \mathrm{~h}$ vigorous activity) or BMI $\left(<30.0 \mathrm{vs} \geq 30.0 \mathrm{~kg} / \mathrm{m}^{2}\right)$. We used likelihood ratio tests to examine whether the association between sitting time and diabetes risk differed by leisure-time physical activity or BMI.

In a first sensitivity analysis, we refrained from using glucose and $\mathrm{HbA}_{1 \mathrm{c}}$ measurements at the additional diabetes examination to classify diabetes at follow-up, as these measurements were performed among people with FINDRISC $\geq 15$ only. Second, we additionally adjusted for self-reported chronic mobility impairment and smoking habits at baseline. Third, because prevalent, symptomatic but undiagnosed diabetes could have influenced sitting time at baseline, we started follow-up 2 years after baseline, assuming that symptomatic diabetes would most likely be detected within this interval. Fourth, because information on age at diabetes diagnosis was lacking for some individuals, we examined the association between sitting time and diabetes incidence using logistic regression analysis. Fifth, we examined the association of sitting time with diabetes stratified by vigorous leisure-time physical activity. Sixth, we examined the association of sitting time with diabetes using finer BMI strata. Seventh, we examined the association between sitting time as a continuous variable and diabetes risk. Data were analysed using Stata version SE 13.1 for Windows (StataCorp LP, College Station, Texas). The study was approved by the regional committee for medical and health research ethics, and all participants gave written informed consent.

\section{Results}

Table 1 shows baseline characteristics (HUNT2, 1995-1997) for participants who did and did not attend the follow-up examination (HUNT3, 2006-2008), and for otherwise eligible participants who were excluded because of missing information at baseline. Of 28,051 participants who attended the follow-up examination, 1253 (4.5\%) developed diabetes during 309,085 person-years of follow-up. Among them, 925 had their diabetes diagnosed between HUNT2 and HUNT3, and 328 had a new diagnosis of diabetes indicated by glucose or $\mathrm{HbA}_{1 \mathrm{c}}$ measurements in HUNT3.

In the total study population, sitting $\geq 8 \mathrm{~h} /$ day was associated with a $17 \%(95 \%$ CI 2,34$)$ higher risk of developing diabetes compared with sitting $\leq 4 \mathrm{~h} /$ day, adjusted for age, sex and education. However, the association was attenuated to a non-significant $9 \%(95 \% \mathrm{CI}-5,26)$ increase in risk after adjustment for BMI and leisure-time physical activity (Table 2), and this attenuation was largely due to adjustment for BMI (Table 3). The association did not substantially differ between women and men (ESM Table 1).

The association between sitting time and diabetes risk differed by baseline leisure-time physical activity level $\left(p_{\text {Interaction }}=0.01\right)$. Among participants with low leisure-time physical activity, sitting 5-7 h/day and $\geq 8 \mathrm{~h} /$ day were associated with a $26 \%(95 \%$ CI 2, 57) and $30 \%$ (95\% CI 5, 61) higher risk of diabetes, respectively, compared with sitting $\leq 4 \mathrm{~h} /$ day. There was no corresponding association among participants with high leisuretime physical activity (Table 2). There was no statistical evidence that the association between sitting time and diabetes risk differed by obesity status $\left(p_{\text {Interaction }}=0.65\right.$; Table 2$)$.

The sensitivity analysis in which glucose and $\mathrm{HbA}_{1 \mathrm{c}}$ measurements at the additional diabetes examination among people with FINDRISC $\geq 15$ was not used for classifying diabetes at follow-up, yielded results similar to the main analysis (ESM Table 2). Adjustment for self-reported chronic mobility impairment (ESM Table 3) or smoking habits (ESM Table 4) had minimal influence on the estimates. Results similar to the main analyses were obtained when we started follow-up 2 years after baseline (ESM Table 5), analysed the association between sitting time and diabetes using logistic regression analysis (ESM Table 6), stratified by vigorous leisure-time physical activity (ESM Table 7) and by finer BMI strata (ESM Table 8), and analysed sitting time as a continuous variable (ESM Table 9).

\section{Discussion}

In this longitudinal population-based study, there was little association between total daily sitting time and diabetes risk in the population as a whole; sitting $\geq 8 \mathrm{~h} /$ day was associated with a modest $17 \%$ risk increase for diabetes that was substantially attenuated after adjustment for BMI. However, among participants with little leisure-time physical activity, sitting $\geq 5 \mathrm{~h} /$ day was associated with a significant $\sim 30 \%$ increased incidence of diabetes. 
Table 1 Baseline characteristics of the 40,825 participants eligible at baseline in 1995-1997 (HUNT2), displayed separately for participants who did and did not attend the follow-up examination in 2006-2008
(HUNT3), as well as baseline characteristics of 12,459 otherwise eligible participants who were excluded because of missing information at baseline

\begin{tabular}{|c|c|c|c|}
\hline Characteristic & $\begin{array}{l}\text { Participants who attended the } \\
\text { follow-up examination }(n=28,051)\end{array}$ & $\begin{array}{l}\text { Participants who did not } \\
\text { attend the follow-up } \\
\text { examination }(n=12,774)\end{array}$ & $\begin{array}{l}\text { Otherwise eligible participants } \\
\text { excluded because of missing } \\
\text { baseline information }(n=12,459)^{\mathrm{a}}\end{array}$ \\
\hline Age (years) & $45(12)$ & $42(15)$ & $46(14)$ \\
\hline Sex (\% women) & 55 & 49 & 51 \\
\hline Sitting time (h/day) & $6.6(3.3)$ & $6.8(3.4)$ & NA \\
\hline \multicolumn{4}{|l|}{ Education $(\%)$} \\
\hline$<10$ years & 28 & 29 & 37 \\
\hline $10-12$ years & 48 & 48 & 46 \\
\hline$>12$ years & 24 & 23 & 17 \\
\hline BMI $\left(\mathrm{kg} / \mathrm{m}^{2}\right)$ & $26.1(3.8)$ & $26.2(4.3)$ & $26.3(4.1)$ \\
\hline \multicolumn{4}{|l|}{ Leisure-time physical activity $(\%)^{\mathrm{b}}$} \\
\hline No & 5 & 8 & 9 \\
\hline Low & 30 & 29 & 32 \\
\hline Medium & 35 & 33 & 32 \\
\hline High & 30 & 31 & 27 \\
\hline Random serum glucose (mmol/l) & $5.2(0.9)$ & $5.2(0.9)$ & $5.3(0.9)$ \\
\hline \multicolumn{4}{|l|}{ Smoking $(\%)$} \\
\hline Never & 47 & 40 & 41 \\
\hline Former & 27 & 22 & 23 \\
\hline Current & 26 & 38 & 36 \\
\hline \multicolumn{4}{|l|}{ Chronic mobility impairment (\%) } \\
\hline No & 90 & 87 & 88 \\
\hline Slight & 5 & 6 & 5 \\
\hline Moderate & 3 & 3 & 3 \\
\hline Severe & 2 & 3 & 3 \\
\hline
\end{tabular}

Data are presented as mean (SD) or \%

${ }^{a}$ Of these, 8617 did not return the second questionnaire with sitting time, 3006 returned the second questionnaire but did not report sitting time, 56 did not answer whether they had diabetes and 780 individuals did not report education level

${ }^{\mathrm{b}}$ Self-reported weekly hours of leisure-time physical activity categorised as no, low ( $<3 \mathrm{~h}$ of light and no vigorous activity), medium ( $\geq 3 \mathrm{~h}$ of light or $<1 \mathrm{~h}$ of vigorous activity) or high ( $\geq 1 \mathrm{~h}$ of vigorous activity)

The strengths of this study include the population-based longitudinal design with an 11 year follow-up, large sample size and reasonable response rates. Key baseline characteristics were similar between participants and non-participants of the follow-up examination, which reduces the potential for bias due to loss of follow-up. The self-report of sitting time may be less accurate than objective measures [11] and lead to conservative estimates of the association between sitting and diabetes. A recent crosssectional study reported an association between sedentary time measured using an accelerometer and prevalent type 2 diabetes [12], but longitudinal studies of objectively measured sitting time and diabetes incidence are lacking. Our results remained similar when the additional measurements from people with FINDRISC $\geq 15$ were not used for classifying diabetes, and the lack of systematic testing for diabetes among participants with FINDRISC $<15$ is unlikely to explain the observed associations. We cannot exclude residual confounding, e.g. from dietary habits such as snacking. The relationship between sitting and BMI is plausibly bidirectional, and it is uncertain whether results with or without BMI adjustment are the most valid estimates of a causal association between sitting and diabetes risk.

Our findings suggest that total sitting time has a less pronounced relationship to diabetes incidence compared with earlier studies using television time as a proxy measure for sitting time. Consistent with our results, television time was more strongly than other types of sitting associated with type 2 diabetes incidence in a 6 year follow-up of the Nurses' Health Study [4]. Also, a Danish cohort study observed a moderate $35 \%$ risk increase of any diabetes associated with total sitting $\geq 10 \mathrm{~h} /$ day compared with $<6 \mathrm{~h} /$ day, but the association was largely attenuated after adjustment for physical activity and BMI [6].

Although sitting time appears to be of little independent importance for diabetes incidence in the population as a whole, prolonged sitting time may contribute to diabetes risk in 
Table 2 HRs for diabetes according to daily sitting time at baseline, overall and stratified by baseline levels of leisure-time physical activity ${ }^{\mathrm{a}}$ and $\mathrm{BMI}^{\mathrm{b}}$, among 28,051 participants of HUNT2 and HUNT3

\begin{tabular}{|c|c|c|c|c|c|}
\hline \multirow[t]{2}{*}{ Sitting time, $\mathrm{h} /$ day } & \multirow[t]{2}{*}{ Number of cases / person-years } & \multicolumn{2}{|c|}{ Model $1^{\mathrm{c}}$} & \multicolumn{2}{|c|}{ Model $2^{\mathrm{d}}$} \\
\hline & & HR & $95 \% \mathrm{CI}$ & HR & $95 \% \mathrm{CI}$ \\
\hline \multicolumn{6}{|c|}{ Total study population } \\
\hline$\leq 4$ & $388 / 99,521$ & 1.00 & Reference & 1.00 & Reference \\
\hline $5-7$ & $404 / 96,685$ & 1.06 & $0.92,1.22$ & 1.00 & $0.86,1.15$ \\
\hline$\geq 8$ & $461 / 112,879$ & 1.17 & $1.02,1.34$ & 1.09 & $0.95,1.26$ \\
\hline \multicolumn{6}{|c|}{ High leisure-time physical activity level ${ }^{\mathrm{e}}$} \\
\hline$\leq 4$ & $207 / 59,351$ & 1.00 & Reference & 1.00 & Reference \\
\hline $5-7$ & $191 / 60,488$ & 0.89 & $0.73,1.09$ & 0.82 & $0.67,1.00$ \\
\hline$\geq 8$ & $236 / 74,293$ & 1.01 & $0.83,1.23$ & 0.95 & $0.79,1.16$ \\
\hline \multicolumn{6}{|c|}{ Low leisure-time physical activity level ${ }^{\mathrm{f}}$} \\
\hline$\leq 4$ & $148 / 35,252$ & 1.00 & Reference & 1.00 & Reference \\
\hline $5-7$ & $193 / 32,806$ & 1.33 & $1.07,1.65$ & 1.26 & $1.02,1.57$ \\
\hline$\geq 8$ & $209 / 36,653$ & 1.38 & $1.11,1.71$ & 1.30 & $1.05,1.61$ \\
\hline \multicolumn{6}{|l|}{$\mathrm{BMI}<30.0 \mathrm{~kg} / \mathrm{m}^{2}$} \\
\hline$\leq 4$ & $212 / 86,390$ & 1.00 & Reference & 1.00 & Reference \\
\hline $5-7$ & $231 / 83,729$ & 1.10 & $0.91,1.33$ & 1.11 & $0.91,1.35$ \\
\hline$\geq 8$ & $259 / 98,309$ & 1.21 & $1.01,1.46$ & 1.19 & $0.98,1.45$ \\
\hline \multicolumn{6}{|l|}{$\mathrm{BMI} \geq 30.0 \mathrm{~kg} / \mathrm{m}^{2}$} \\
\hline$\leq 4$ & $176 / 12,960$ & 1.00 & Reference & 1.00 & Reference \\
\hline $5-7$ & $173 / 12,863$ & 0.97 & $0.79,1.20$ & 0.92 & $0.74,1.15$ \\
\hline$\geq 8$ & 199 / 14,209 & 1.04 & $0.84,1.28$ & 0.98 & $0.79,1.21$ \\
\hline
\end{tabular}

${ }^{\mathrm{a}} p_{\text {Interaction }}=0.009$ between sitting time and leisure-time physical activity in model adjusted for age, sex and education; $p_{\text {Interaction }}=0.01$ between sitting time and leisure-time physical activity in model additionally adjusted for BMI

${ }^{\mathrm{b}} p_{\text {Interaction }}=0.66$ between sitting time and BMI in model adjusted for age, sex and education; $p_{\text {Interaction }}=0.65$ between sitting time and BMI in model additionally adjusted for leisure-time physical activity

${ }^{\mathrm{c}}$ Adjusted for age, sex and education

${ }^{\mathrm{d}}$ Adjusted for age, sex, education, leisure-time physical activity and BMI. A total of 987 (3.5\%) participants were excluded because of missing information on physical activity or BMI

${ }^{\mathrm{e}} \geq 3 \mathrm{~h}$ light or $>0 \mathrm{~h}$ vigorous leisure-time physical activity per week

${ }^{\mathrm{f}} \leq 2 \mathrm{~h}$ light and no vigorous leisure-time physical activity per week subgroups of the population. The Danish cohort study suggested that sedentary time is associated with increased diabetes risk among inactive or obese people [6]. Our results are also consistent with those of meta-analyses of sedentary time and all-cause mortality, which indicate that prolonged sitting may have adverse effects particularly among inactive people [2, 13].

Table 3 The influence of BMI and leisure-time physical activity adjustments on the HRs for diabetes according to daily sitting time at baseline, among 28,051 participants of HUNT2 and HUNT3

\begin{tabular}{|c|c|c|c|c|c|c|}
\hline \multirow[t]{2}{*}{ Adjustment variables } & \multicolumn{2}{|c|}{ Sitting time $\leq 4 \mathrm{~h} /$ day } & \multicolumn{2}{|c|}{ Sitting time $5-7 \mathrm{~h} /$ day } & \multicolumn{2}{|c|}{ Sitting time $\geq 8 \mathrm{~h} /$ day } \\
\hline & HR & $95 \% \mathrm{CI}$ & HR & $95 \% \mathrm{CI}$ & HR & $95 \% \mathrm{CI}$ \\
\hline Age, sex, and education & 1.00 & Reference & 1.06 & $0.92,1.22$ & 1.17 & $1.02,1.34$ \\
\hline Age, sex, education and $\mathrm{BMI}^{\mathrm{a}}$ & 1.00 & Reference & 0.99 & $0.86,1.14$ & 1.11 & $0.97,1.28$ \\
\hline Age, sex, education and leisure-time physical activity ${ }^{\mathrm{b}}$ & 1.00 & Reference & 1.07 & $0.92,1.24$ & 1.15 & $1.00,1.33$ \\
\hline Age, sex, education and vigorous leisure-time physical activity ${ }^{c}$ & 1.00 & Reference & 1.06 & $0.92,1.23$ & 1.15 & $0.99,1.32$ \\
\hline
\end{tabular}

${ }^{\mathrm{a}} \mathrm{A}$ total of 57 (0.2\%) participants were excluded because of missing information on BMI

${ }^{\mathrm{b}} \mathrm{A}$ total of 931 (3.3\%) participants were excluded because of missing information on physical activity

${ }^{\mathrm{c}}$ Adjusted for weekly hours of vigorous leisure-time physical activity categorised as no, $<1,1-2$ or $\geq 3$, as an alternative adjustment for leisure-time physical activity. A total of 931 (3.3\%) participants were excluded because of missing information on physical activity 
In conclusion, the results of this longitudinal populationbased study suggest that total daily sitting time has little association with the risk of any diabetes in the population as a whole, but prolonged sitting time may contribute to increased diabetes risk among physically inactive people.

Acknowledgements The HUNT Study is a collaborative effort between the HUNT Research Centre (Faculty of Medicine, NTNU, Norwegian University of Science and Technology), Nord-Trøndelag County Council, Central Norway Health Authority and the Norwegian Institute of Public Health.

Data availability Data from the HUNT Study that is used in research projects will, when reasonably requested by others, be made available on request to the HUNT Data Access Committee (hunt@medisin.ntnu.no). The HUNT data access information (http://www.ntnu.edu/hunt/data) describes the policy regarding data availability in detail.

Funding The study was funded by the Norwegian University of Science and Technology and the Research Council of Norway (231149/F20).

Duality of interest The authors declare that there is no duality of interest associated with this manuscript.

Contribution statement $\mathrm{BO} \AA \mathrm{AR}$ and $\mathrm{AB}$ designed the present study. $\mathrm{KM}$ and SK contributed to acquisition of data. BOA analysed the data and wrote the manuscript together with $\mathrm{AB}$. KM, SK, and VR critically revised the manuscript. All authors interpreted the data and approved the final version of the manuscript. $\mathrm{BO} \AA$ is the guarantor of this work.

\section{References}

1. Henson J, Dunstan DW, Davies MJ, Yates T (2016) Sedentary behaviour as a new behavioural target in the prevention and treatment of type 2 diabetes. Diabetes Metab Res Rev 32(Suppl 1):213-220
2. Biswas A, Oh PI, Faulkner GE et al (2015) Sedentary time and its association with risk for disease incidence, mortality, and hospitalization in adults: a systematic review and meta-analysis. Ann Intern Med 162:123-132

3. Grøntved A, Hu FB (2011) Television viewing and risk of type 2 diabetes, cardiovascular disease, and all-cause mortality: a metaanalysis. JAMA 305:2448-2455

4. Hu FB, Li TY, Colditz GA, Willett WC, Manson JE (2003) Television watching and other sedentary behaviors in relation to risk of obesity and type 2 diabetes mellitus in women. JAMA 289:1785-1791

5. Sisson SB, Broyles ST (2012) Social-ecological correlates of excessive TV viewing: difference by race and sex. J Phys Act Health 9:449-455

6. Petersen CB, Bauman A, Tolstrup JS (2016) Total sitting time and the risk of incident diabetes in Danish adults (the DANHES cohort) over 5 years: a prospective study. Br J Sports Med 50: 1382-1387

7. Krokstad S, Langhammer A, Hveem K et al (2013) Cohort profile: the HUNT Study, Norway. Int J Epidemiol 42:968-977

8. Jølle A, Midthjell K, Holmen J et al (2016) Impact of sex and age on the performance of FINDRISC: the HUNT Study in Norway. BMJ Open Diabetes Res Care 4:e000217

9. Midthjell K, Holmen J, Bjørndal A, Lund-Larsen G (1992) Is questionnaire information valid in the study of a chronic disease such as diabetes? The Nord-Trøndelag diabetes study. J Epidemiol Community Health 46:537-542

10. Kurtze N, Rangul V, Hustvedt BE, Flanders WD (2007) Reliability and validity of self-reported physical activity in the Nord-Trøndelag Health Study (HUNT2). Eur J Epidemiol 22:379-387

11. Rosenberg DE, Bull FC, Marshall AL, Sallis JF, Bauman AE (2008) Assessment of sedentary behavior with the International Physical Activity Questionnaire. J Phys Act Health 5(Suppl 1): S30-S44

12. van der Berg JD, Stehouwer CD, Bosma H et al (2016) Associations of total amount and patterns of sedentary behaviour with type 2 diabetes and the metabolic syndrome: the Maastricht Study. Diabetologia 59:709-718

13. Ekelund U, Steene-Johannessen J, Brown WJ et al (2016) Does physical activity attenuate, or even eliminate, the detrimental association of sitting time with mortality? A harmonised meta-analysis of data from more than 1 million men and women. Lancet 388: 1302-1310 